\title{
Phase relationships in presence of a synthetic large-scale in a turbulent boundary layer
}

\author{
Subrahmanyam Duvvuri ${ }^{\dagger}$ and Beverley J. McKeon $\S$ \\ Graduate Aerospace Laboratories, California Institute of Technology, Pasadena, CA 91125
}

\begin{abstract}
A synthetic spanwise-constant spatio-temporal mode is excited in a flat plate turbulent boundary layer through a spatially impulsive patch of dynamic wall-roughness. The streamwise wavelength of the synthetic mode approximately corresponds to the very large-scale motions present in high Reynolds number wall turbulence. Hot wire anemometer measurements made downstream of the roughness forcing reveal the nature of the two dimensional synthetic large-scale and its influence on the small-scale turbulence. A clear phase organizing effect on the small-scales is noticed in presence of the synthetic large-scale. A thorough understanding of these phase relations lays the foundation for a framework that allows for practical manipulation of energetic small-scale turbulence through large-scale inputs by utilizing the inherent non-linear coupling present in the governing Navier-Stokes equations.
\end{abstract}

\section{Introduction}

The presence of very large-scale motions in wall-bounded turbulent flows has been confirmed by several investigators through experimental and numerical studies in the past two decades (see Smits et al ${ }^{1}$ for a recent review of high Reynolds number wall turbulence). These coherent motions, referred to as VLSMs, are centered in the logarithmic region and extend several boundary layer thicknesses (or pipe radii) in the streamwise direction. VLSMs become energetically significant and extend their footprint down to the wall with increasing Reynolds numbers. The influence of VLSMs on the near-wall cycle of turbulence is of particular interest from a scientific and an engineering perspective. Hutchins and Marusic ${ }^{2}$ suggest a modulation effect on the small-scale energy due to large-scale motions, analogous to a modulating signal. This effect is quantified in Mathis $e t a l^{3}$ by a correlation coefficient between large-scale activity and the largescale envelope of small-scales, termed as amplitude modulation coefficient, in canonical turbulent boundary layer flows over a wide Reynolds number range.

An attempt to generalize the influence of large-scale motions on small-scale turbulence activity is made in the present experimental study by introducing a synthetic large-scale into a boundary layer flow. Recent studies by Jacobi and McKeon ${ }^{4,5}$ thoroughly characterize the effects of a spatially impulsive patch of static and dynamic $k$-type wall-roughness in a boundary layer flow. The experimental technique of dynamic wallroughness described in Jacobi and $\mathrm{McKeon}^{5}$ is seen to be very effective in forcing a spatio-temporal mode in wall-bounded flows. The same technique is used here with a modified roughness geometry and forcing frequency to excite a large-scale two-dimensional mode with zero spanwise wavenumber, and study its effects on large- and small-scale phase relationships.

A description of the experiment is presented in section II; a set of hot-wire measurements with dynamic roughness forcing and a set of canonical flow measurements are made. Results from the experiments are discussed in section III, followed by a summary and concluding remarks in section IV.

${ }^{\dagger} \mathrm{PhD}$ Candidate in Aeronautics, AIAA Student Member.

$\S$ Professor of Aeronautics, AIAA Associate Fellow. 


\section{Experimental method}

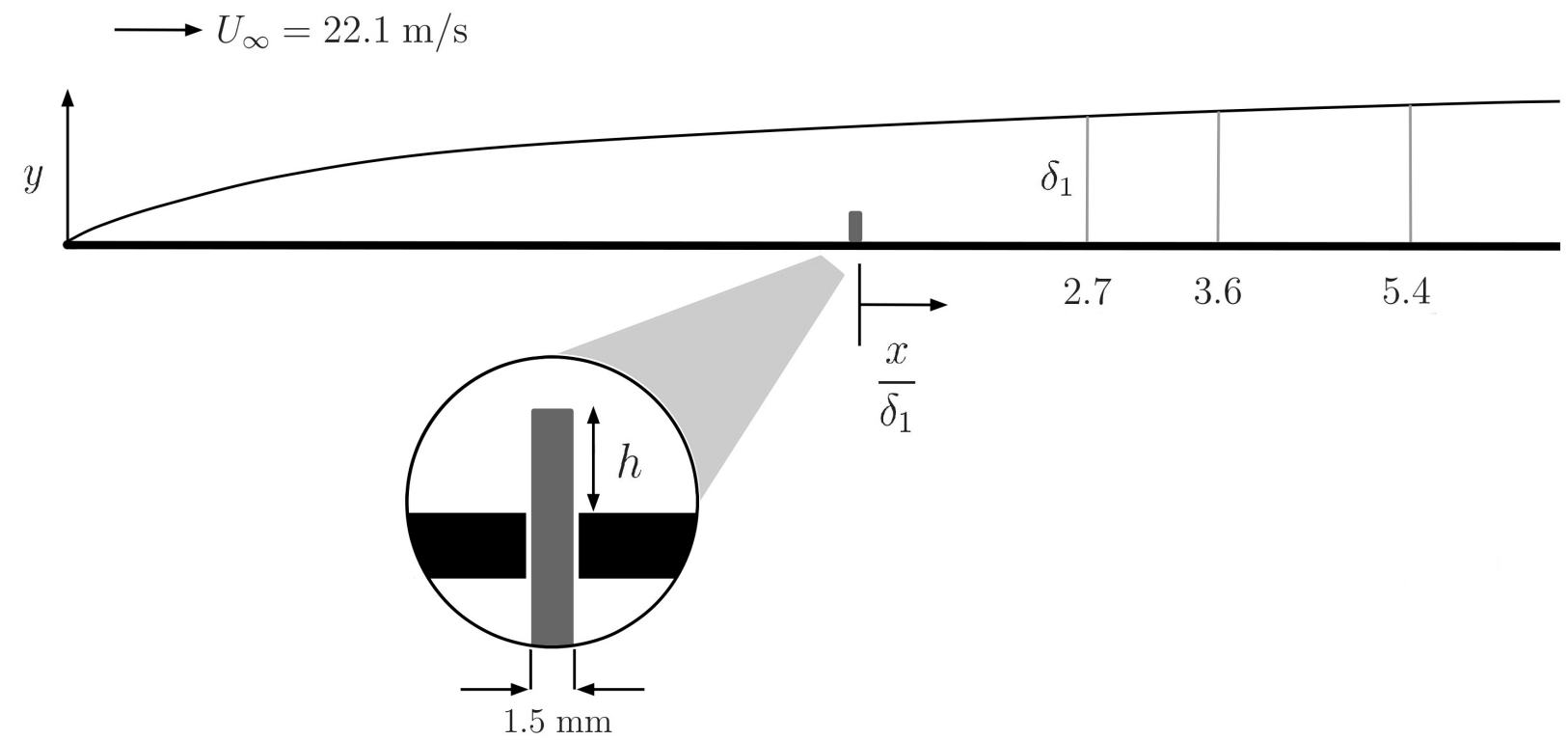

Figure 1. Schematic shows the dynamic single-rib roughness perturbation in a flat plate boundary layer flow (not to scale). Frequency of roughness oscillations $(f)$ is set to $50 \mathrm{~Hz}$ with an amplitude $(h)$ of $h_{\mathrm{rms}}=0.54 \mathrm{~mm}$. Downstream hot-wire measurement locations are also marked, see table 1 for other details.

A zero-pressure-gradient turbulent boundary layer flow over a smooth flat plate was set up for this study in the Merrill wind tunnel at Caltech. Earlier experiments by Jacobi and McKeon ${ }^{4,5}$ were performed in the same facility and a full description of a similar set-up can also be found there. The tunnel test section has an area of $2 \mathrm{ft} \times 2 \mathrm{ft}$ and is $8 \mathrm{ft}$ in length, and has an adjustable height ceiling. Root-mean-square variation in pressure coefficient $\left(C_{p}\right)_{\mathrm{rms}}$ over the spatial extent of measurement locations is less than $1 \%$ at a free-stream velocity of $U_{\infty}=22.1 \mathrm{~m} / \mathrm{s}$ used in all experiments. The plate, constructed from acrylic (PMMA), consists of an elliptic leading edge with a $0.76 \mathrm{~mm}$ diameter wire glued to its surface $19 \mathrm{~mm}$ downstream of the tip to serve as a trip for flow transition. A slotted insert fitted into the plate downstream of the trip allows for a spatially impulsive two dimensional wall-roughness in the form of a thin straight aluminum rib aligned along the spanwise direction to protrude into the flow from beneath (see figure 1). The insert slot is machined with sufficient tolerance to allow rib reciprocation with minimal friction but prevent bleeding of air between the plate surfaces. The rib has a spanwise extent of $230 \mathrm{~mm}\left(\approx 14 \delta_{1}\right.$, see table 1$)$ and is positioned at a downstream location of $890 \mathrm{~mm}$ from the trip wire (the boundary layer is fully turbulent at this location with $R e_{\theta} \approx 2750$ ).

This rib is connected to a crank shaft mechanism and DC motor positioned underneath the test section such that the motor shaft rotations translate to linear oscillations of the rib in the vertical direction. At the trough of each oscillation cycle, the rib sits flush with the plate surface. The frequency of oscillations is controlled by the motor rotation rate, and amplitude is fixed by an offset between the connecting rod and the shaft center. This offset was nominally set to $0.3 \mathrm{~mm}$, resulting in a root-mean-square amplitude of $0.6 / \sqrt{2}$ $\mathrm{mm}$ at low oscillation frequencies. However, at the operation frequency of $f=50 \mathrm{~Hz}$ used in this study, slippage due to wear and heating resulted in a root-mean-square amplitude of $h_{r m s}=0.54 \pm 0.02 \mathrm{~mm}$. A magnetic linear encoder (Renishaw LM10) with a resolution of $1 \mu \mathrm{m}$ is attached to the actuation mechanism to record time-resolved input signal to the flow, i.e., perturbation height $h(t)$.

In the experiments of Jacobi and McKeon, ${ }^{5}$ a $k$-type roughness comprising of four parallel ribs aligned along the spanwise direction with an inter-rib separation of $6.35 \mathrm{~mm}$ and $h_{r m s}=1.16 \mathrm{~mm}$ was used. In comparison, the roughness used here has a shorter streamwise extent and a smaller amplitude. Also, the forcing frequency of the present study is higher compared to $f=30 \mathrm{~Hz}$ of Jacobi and McKeon. ${ }^{5}$ 
Hot-wire measurements were made at three different locations downstream of the roughness (see table 1) using a Dantec 55P05 boundary layer probe (5 $\mu \mathrm{m}$ diameter and $1.25 \mathrm{~mm}$ active length) with a Dantec StreamLine Pro anemometer. This system was calibrated in situ against Pitot probe measurements made using a Baratron MKS 220DD pressure transducer (0-20 Torr range). The system's frequency response was estimated through a square-wave test to be $\approx 25 \mathrm{kHz}$ and $\approx 55 \mathrm{kHz}$ at mean velocities of $0 \mathrm{~m} / \mathrm{s}$ and $22.1 \mathrm{~m} / \mathrm{s}$ respectively. The hot-wire probe holder is affixed to a stepper motor driven vertical traverse $(2.5 \mu \mathrm{m} /$ rotation) controlled by a computer. An external magnetic linear encoder (Renishaw LMA10) of resolution $1 \mu \mathrm{m}$ is attached to the traverse to provide a secondary check on the probe height from the wall. For each boundary layer traverse, the nearest position to the wall was set manually by calibrating the height with a macro lens camera. The probe was subsequently moved automatically in preset steps to capture the velocity profile in 40 logarithmically spaced segments.

Roughness encoder and anemometer data were synchronously sampled (phase-locked) at $60 \mathrm{kHz}$ by a National Instruments data acquisition computer card (NI-6154). The hot-wire signal was filtered prior to acquisition by a Dantec built-in third order low-pass Butterworth (linear phase) filter with a cut-off set to 30 $\mathrm{kHz}$. At each step, data was recorded for 50 seconds, corresponding to $\approx 65000$ outer eddy turnover periods.

\section{Results and discussion}

\begin{tabular}{|c|c|c|c|c|}
\hline Location & $x / \delta_{1}$ & $\delta(\mathrm{mm})$ & $R e_{\theta}$ & $R e_{\tau}$ \\
\hline 1 & 2.7 & 16.55 & 2780 & 940 \\
2 & 3.6 & 16.64 & 2804 & 942 \\
3 & 5.4 & 16.75 & 2892 & 955 \\
\hline
\end{tabular}

Table 1. The Canonical (smooth wall) boundary layer conditions shown for three measurement locations downstream of perturbation. $\delta_{1}$ is the layer thickness $\delta\left(99 \%\right.$ of $\left.U_{\infty}\right)$ at location 1 . Karman number $\left(R e_{\tau}\right)$ is estimated is using the Coles-Fernholz relation (see text).
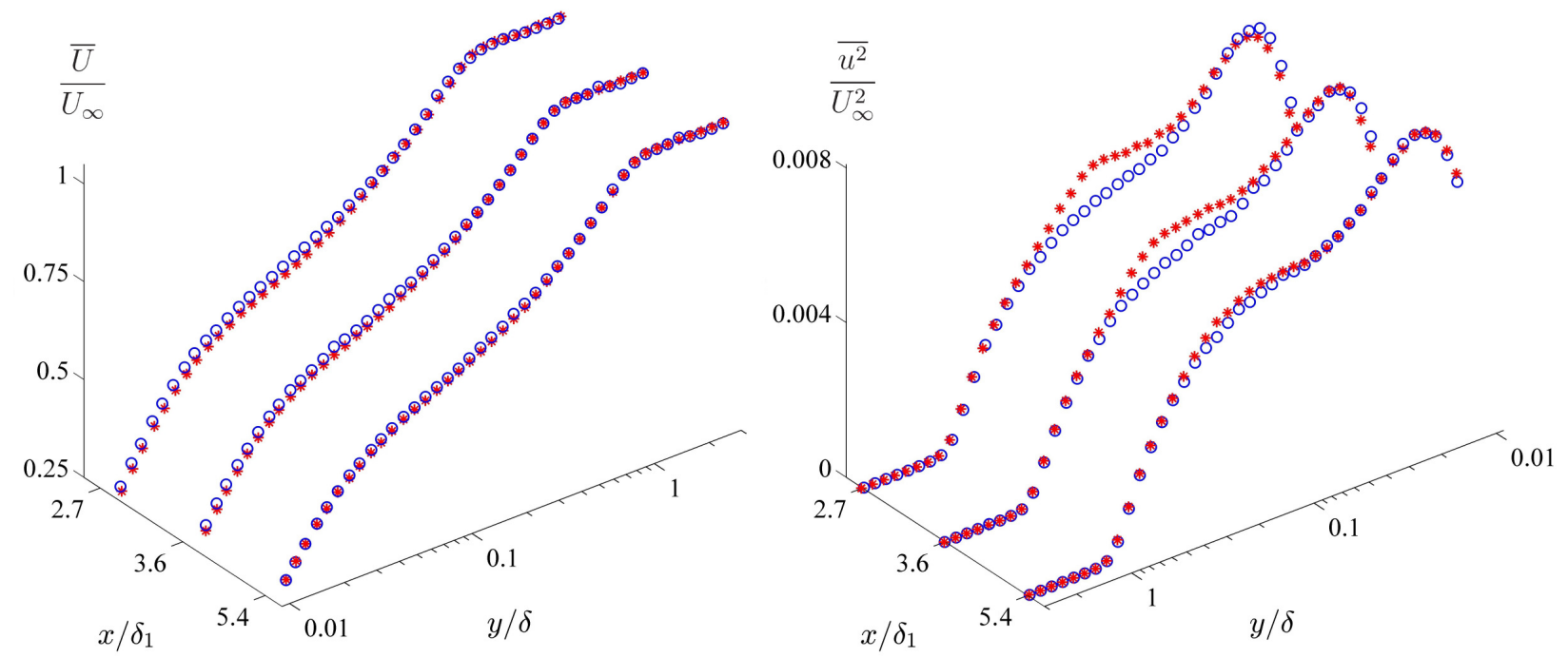

Figure 2. A comparison of the canonical and the turbulent boundary layer: (left) streamwise mean velocity profiles; (right) streamwise turbulence intensity profiles. Plot legend: $\circ$, canonical flow; $*$, forced flow.

Relevant flow properties for the canonical boundary layer at the three measurement locations (marked in figure 1) are shown in table 1 . Karman numbers $\left(R e_{\tau}\right)$ shown in the table's last column are estimated from local momentum thickness Reynolds numbers $\left(R e_{\theta}\right)$ using the Coles-Fernholz empirical relation

$$
C_{f}=\frac{2}{\left[\frac{1}{\kappa} \log \left(R e_{\theta}\right)+C\right]^{2}}
$$


where $C_{f}$ is the skin-friction coefficient. The Karman constant $\kappa$ and $C$ in the above relation are taken to be $\kappa=0.384, C=4.127$ following Nagib et al. ${ }^{6}$ Based on these estimates, boundary layer heights scaled in inner units $\left(y^{+}\right)$are shown for reference along with outer scaling $(y / \delta)$ on some figures in this section. From the expected location of the near-wall peak in turbulence intensity of $y^{+}=15$ (e.g., see figure 7 in DeGraaf and Eaton ${ }^{7}$ ), these estimates are found to be accurate to within $5 y^{+}$units. However, all velocity and spectral energy information presented here is scaled only in outer units given the uncertainty in friction velocity.
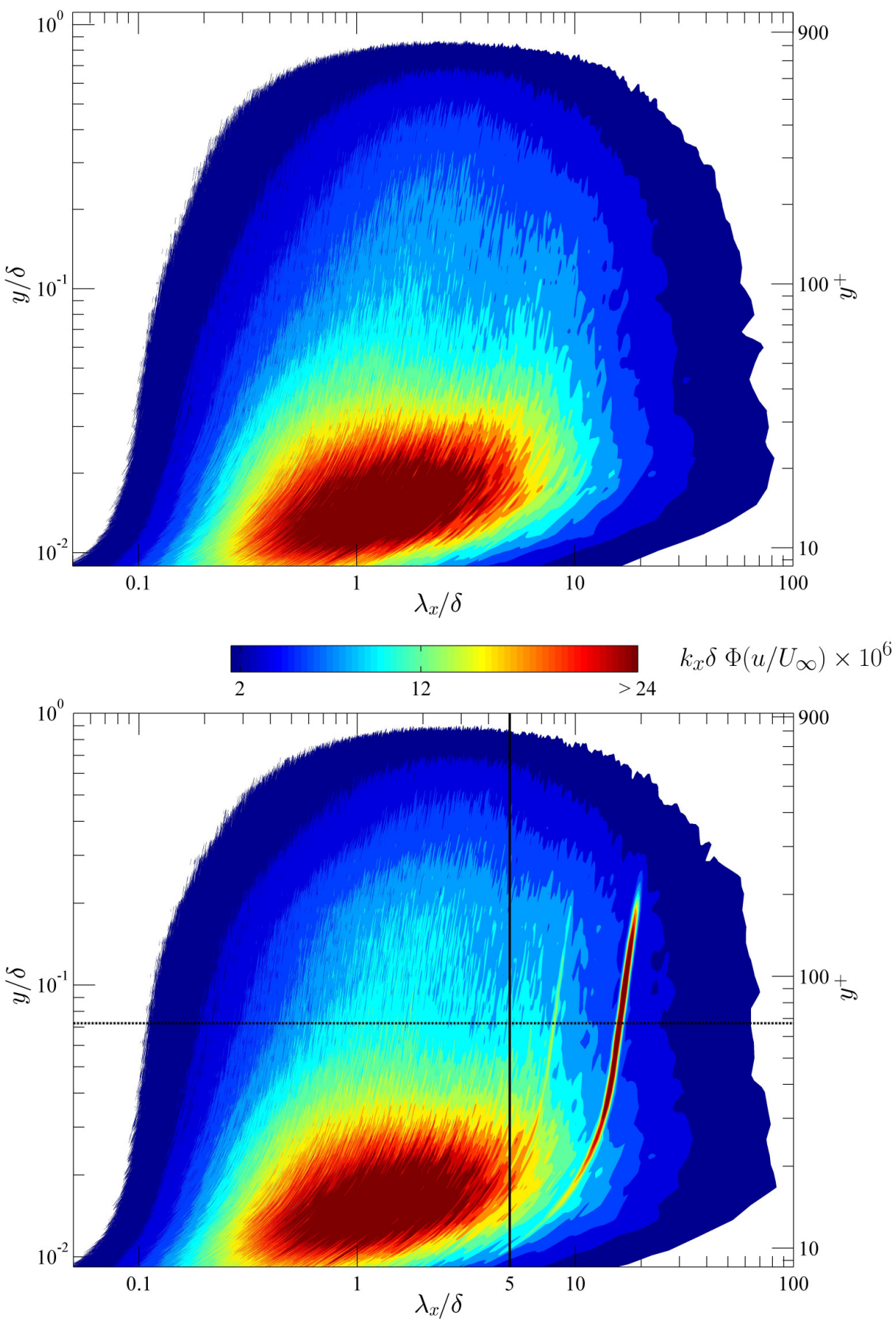

Figure 3. Pre-multiplied and scaled power spectral density $k_{x} \delta \Phi\left(u / U_{\infty}\right) \times 10^{6}$ at $x / \delta_{1}=2.7$ for the canonical (top) and the forced boundary layer (bottom). Color levels are held fixed for both plots. The synthetic large-scale signature can be seen in the bottom plot as a thin energetic streak centered around $\lambda_{x} \approx 15 \delta$ and extending from $y / \delta \approx 0.01$ to $y / \delta \approx 0.3$. Filter cut-off at $\lambda_{x}=5 \delta$ for large- and small-scale separation is shown by a vertical line in the same plot. The critical layer location (see section III B) for the synthetic mode is also marked by a dashed horizontal line. 

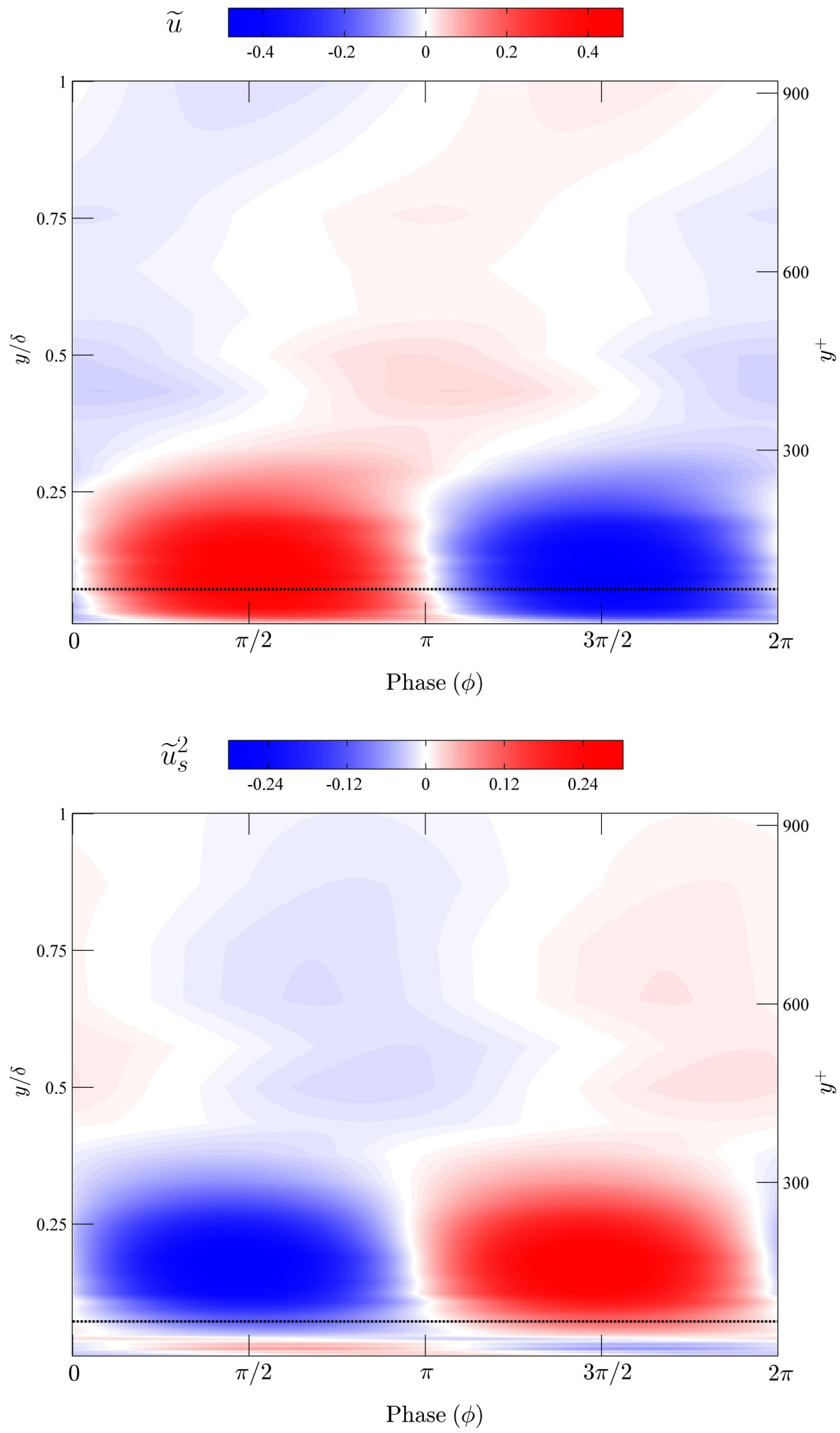

Figure 4. Phasemaps show $\tilde{u}$ (top) and $\widetilde{u}_{s}^{2}$ (bottom) over one temporal period shown at $x / \delta_{1}=2.7$ for the forced boundary layer. The critical layer $\left(y_{c}\right)$ is marked by a dashed horizontal line in both the plots (see section III B).

$$
5 \text { of } 10
$$




\section{III.A. Statistical and spectral quantities}

Velocity data $U(y, t)$ from the hot-wire measurements is decomposed into time mean $\bar{U}(y)$ and fluctuating $u(y, t)$ components (i.e., a Reynolds decomposition). Profiles of mean velocity $\bar{U}(y)$ and variance of turbulent fluctuations $\overline{u^{2}}(y)$ are shown in figure 2. No discernible difference in mean velocity, and hence the mean shear stress, can be seen between the canonical and the forced flow. A small bump in energy due to forcing is seen around $y / \delta=0.1$ in the variance profile at $x / \delta_{1}=2.7$; it begins to disappear further downstream at $x / \delta_{1}=3.6$ and 5.4 with decay of energy imparted by the disturbance at $x / \delta_{1}=0$. Any static roughness effects in the flow as described in Jacobi and $\mathrm{McKeon}^{4}$ are minimal at locations investigated here; this is to be expected considering the relatively weaker nature of the roughness perturbation. Dynamic forcing effects are still present in the flow as evidenced by the clear spectral signature of a synthetic large-scale in the power spectra $(\Phi)$ at location $x / \delta_{1}=2.7$ shown in figure 3. Taylor's hypothesis of frozen turbulence is used to translate temporal scales to spatial length scales in the streamwise direction $(x)$ using the local mean velocity $\bar{U}(y)$. For purposes of this analysis, scales with streamwise wavelength $\left(\lambda_{x}=2 \pi / k_{x}\right)$ smaller than $5 \delta$ are referred to as small-scales (region left of the vertical filter line in figure 3 ).

\section{III.B. Synthetic large-scale}

The two dimensional structure of the synthetic large-scale can be recovered from velocity fluctuations $u(y, t)$ by phase-averaging the signal with reference to the input perturbation (obtained from the encoder signal) at each height following Hussain and Reynolds. ${ }^{8}$ The phasemap constructed by this procedure at location $x / \delta_{1}=2.7$ for the forced boundary layer is shown on the top plot in figure 4 . The quantity $\widetilde{u}$, which represents the synthetic large-scale velocity, is obtained by band-pass filtering $u(y, t)$ with a narrow Fourier filter (10 Hz bandwidth) around the forcing frequency and phase-averaging the filter output over 2700 cycles. Such a filtering process mitigates any de-correlating effects caused by frequency content naturally present in the flow. It is noted that by definition $\langle\widetilde{u}\rangle=0$, where \langle\rangle represents the average over one period. Similar phasemaps are constructed at $x / \delta_{1}=3.6$ and 5.4 (not shown here) to estimate the wavelength of the synthetic scale by tracking changes in phase $(\phi)$ with $x$; the streamwise wavelength is found to be $\approx 15 \delta . \widetilde{u}$ is seen to be qualitatively similar to the two-dimensional synthetic modes observed previously in Jacobi and McKeon. ${ }^{5}$ However, this mode is shorter than the synthetic mode studied by Jacobi and McKeon $\left(\lambda_{x}=18.7 \delta\right)$, and closer to the streamwise wavelength of VLSMs $(6 \delta-10 \delta)$ present in wall-bounded flows at higher Reynolds numbers. The wavespeed $(c)$ of the synthetic mode is given by $c=f \lambda_{x}$; with $f=50 \mathrm{~Hz}$ and $\lambda_{x}=15 \delta$, we have $c=0.59 U_{\infty}$. The critical layer location $\left(y_{c}\right)$ for the synthetic mode (i.e., the location in $y$ where $\left.\bar{U}\left(y_{c}\right)=c\right)$ is found to be $y_{c}=0.072 \delta\left(y_{c}^{+} \approx 67\right)$; this is marked by a dashed line in all relevant figures of this section.

\section{III.C. Directly coupled small-scales}

The quadratic nature of non-linear coupling between scales in turbulence (arising from the convective acceleration term in the Navier-Stokes equations) implies that scale interactions must be triadically consistent, i.e., a pair of modes with wavenumbers $k_{1}$ and $k_{2}$ can directly influence (or force) a mode with wavenumber $k_{3}$ only if $k_{3}=k_{1}+k_{2}$ or $k_{3}=k_{1}-k_{2}$. To understand the influence of the synthetic large-scale on small-scales that directly couple to it through such triadic interactions, we study the small-scale velocity signal $\left(u_{s}\right)$ in the following way. Firstly, $u_{s}$ is obtained by short-wavelength-pass filtering the velocity signal $u(y, t)$ through a spatial Fourier filter with a cut-off at $5 \delta$ (indicated in figure 3 bottom plot). $u_{s}$ is then squared and band-pass filtered around the forcing frequency as described earlier for $\widetilde{u}$. The filter output is phase-averaged with reference to the input perturbation over 2700 cycles and mean removed to obtain $\widetilde{u}_{s}^{2}$ such that $\left\langle\widetilde{u}_{s}^{2}\right\rangle=0 . \widetilde{u}_{s}^{2}$ captures the envelope of small-scale activity that is directly coupled to the synthetic large-scale through triadic interactions.

$\widetilde{u}_{s}^{2}$ corresponding to $\tilde{u}$ at $x / \delta_{1}=2.7$ is shown on the bottom plot of figure 4 . From a visual comparison between $\widetilde{u}$ and $\widetilde{u}_{s}^{2}$ (top and bottom plots) in figure 4 , it is seen that the envelope of triadically interacting small-scales is clearly out of phase with the synthetic large-scale between the regions $y / \delta \approx 0.05$ and $y / \delta \approx 0.3$ 
and in phase very near to the wall. The phase difference between them can be quantified by the correlation coefficient $R_{c c}(y)$ defined as

$$
R_{c c}=\frac{\left\langle\widetilde{u} \widetilde{u}_{s}^{2}\right\rangle}{\sqrt{\left\langle(\widetilde{u})^{2}\right\rangle} \sqrt{\left\langle\left(\widetilde{u}_{s}^{2}\right)^{2}\right\rangle}}
$$

This is similar to the amplitude modulation coefficient defined by Mathis et $a l^{3}$ (see next sub-section), with the key difference that $R_{c c}(y)$ is a measure of the modulating influence of the synthetic large-scale on only those small-scales that are directly coupled to it. Chung and $\mathrm{McKeon}^{9}$ note that a correlation between two such quantities can also be directly interpreted as a phase relationship. Jacobi and McKeon ${ }^{10}$ use the same interpretation in their analysis of phase relationships in a canonical turbulent boundary layer.

$R_{c c}(y)$ for $x / \delta_{1}=2.7$ is shown in figure 5. Following the interpretation of Chung and McKeon, ${ }^{9}$ the envelope of triadically interacting small-scales is found to be in phase $\left(R_{c c}=1\right)$ with the synthetic largescale very near to the wall $(y / \delta<0.03)$. A phase jump of $\pi$ radians occurs around $y / \delta \approx 0.04$ and the envelope of triadically interacting small-scales is out of phase $\left(R_{c c}=-1\right)$ with the synthetic large-scale between $y / \delta \approx 0.05$ and $y / \delta \approx 0.3$. Above $y / \delta \approx 0.3$, the envelope of triadically interacting small-scales moves gradually to be back in phase with the synthetic large-scale.

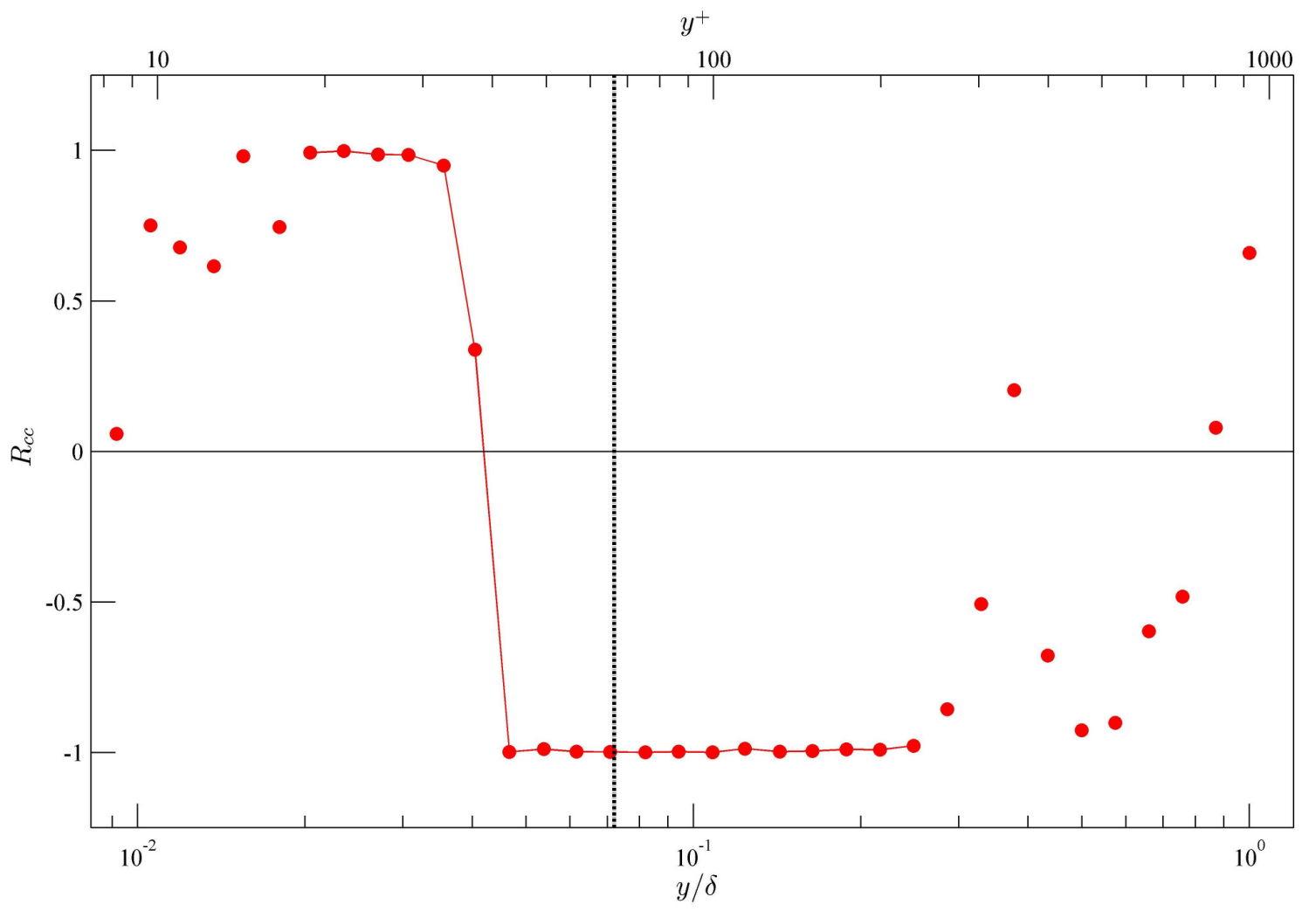

Figure 5. Correlation coefficient $R_{c c}$ between envelope of triadically interacting small-scales and synthetic large-scale. The critical layer $\left(y_{c}\right)$ is marked by a dashed vertical line.

\section{III.D. All large- and small-scales}

The phase relationship between all large-scales and the envelope of all small-scales in the flow can be understood by studying the amplitude modulation coefficient $\left(R_{a m}\right)$ defined by Mathis et $a l^{3}$ as

$$
R_{a m}=\frac{\left\langle u_{l} \mathcal{E}_{l}\left(u_{s}\right)\right\rangle}{\sqrt{\left\langle u_{l}^{2}\right\rangle} \sqrt{\left\langle\mathcal{E}_{l}\left(u_{s}\right)^{2}\right\rangle}}
$$




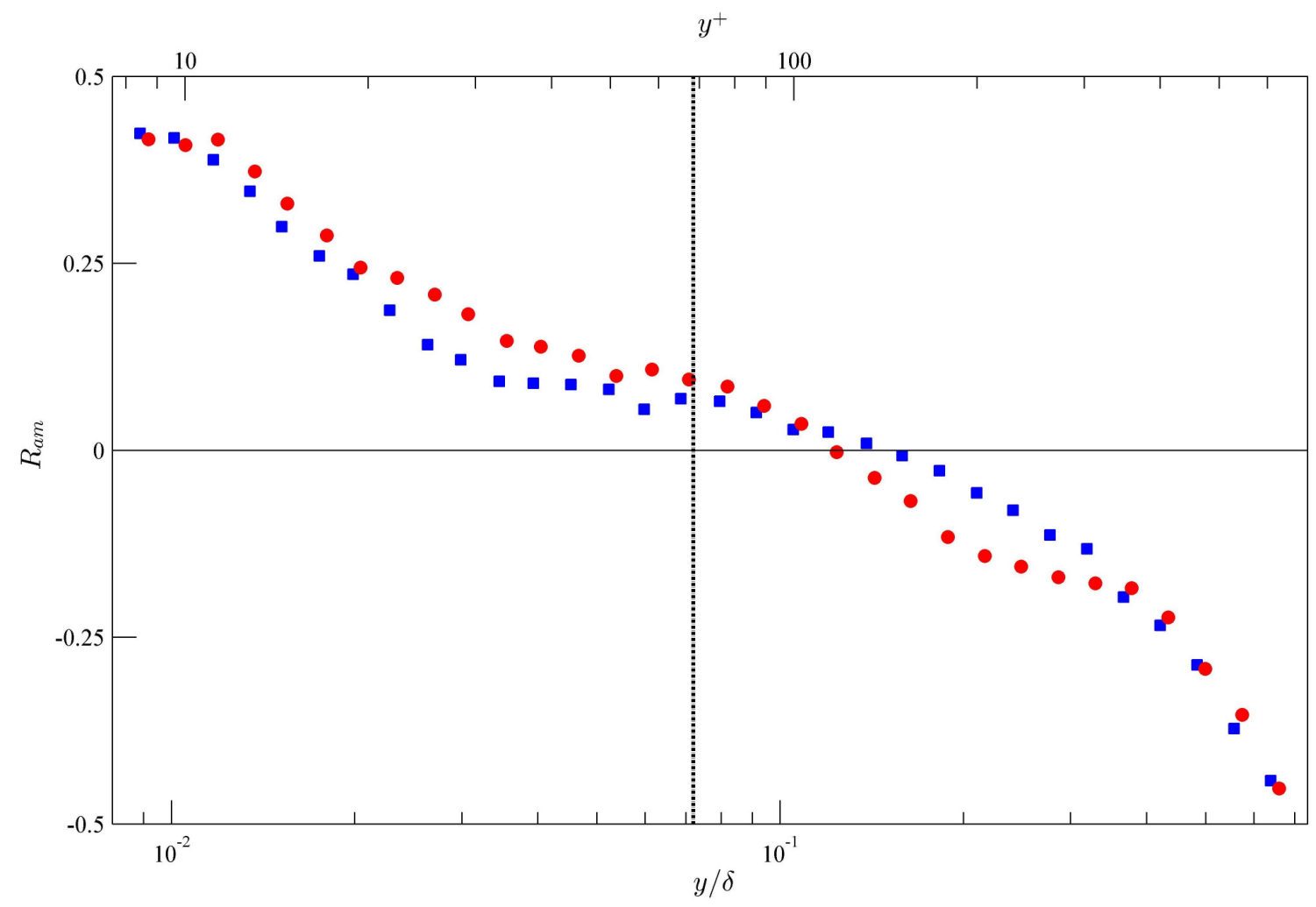

Figure 6. Coefficient of amplitude modulation $R_{a m}$ of the small-scale envelope by large-scales. The critical layer $\left(y_{c}\right)$ is marked by a dashed vertical line. Plot legend: -, canonical flow; •, forced flow.

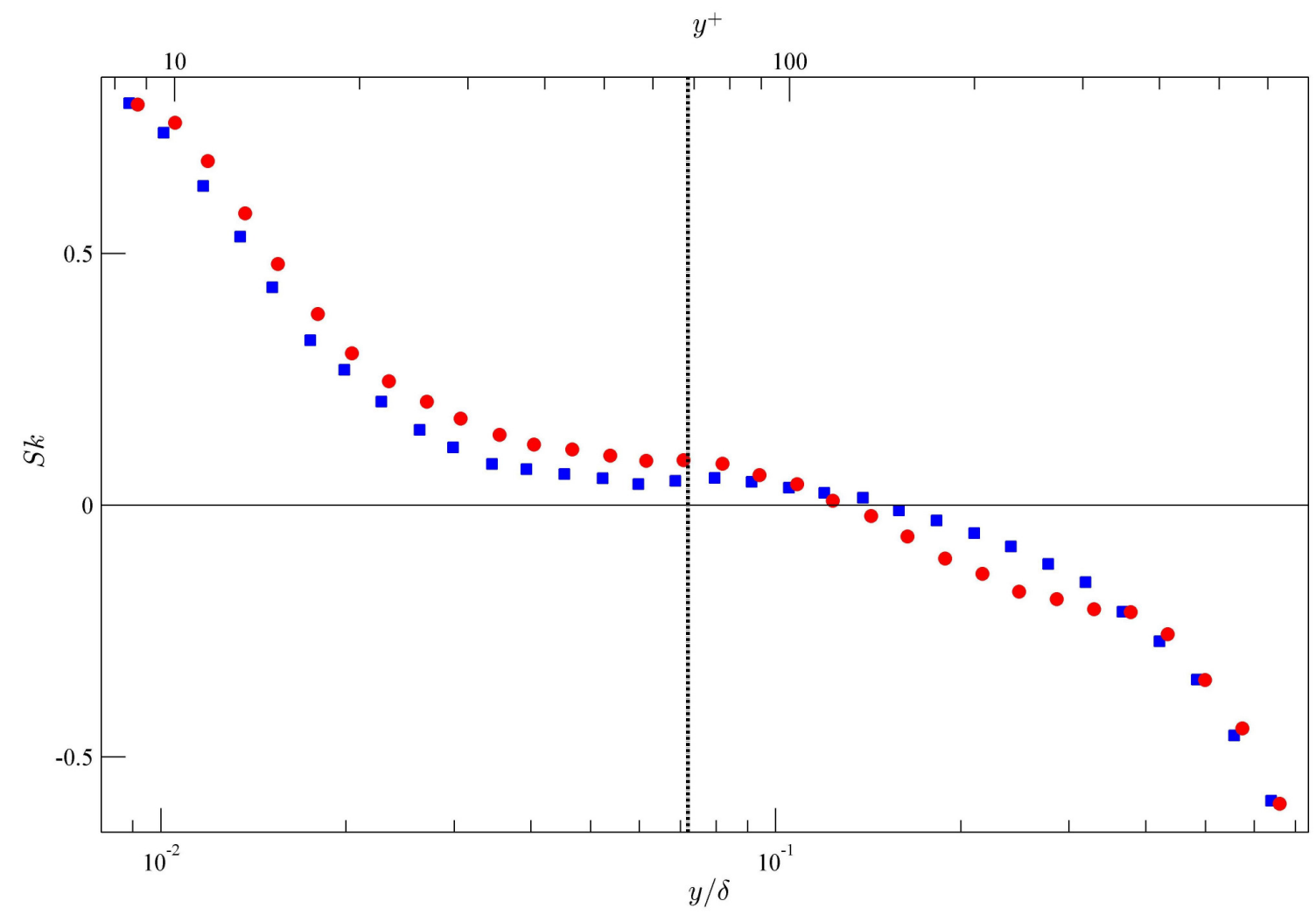

Figure 7. Skewness profiles of velocity fluctuations. The critical layer $\left(y_{c}\right)$ is marked by a dashed vertical line. Plot legend: •, canonical flow; •, forced flow. 
Here $u_{l}\left(=u-u_{s}\right)$ is obtained by long-wavelength-pass filtering $u$ through a spatial Fourier filter with cut-off at $5 \delta . \mathcal{E}_{l}\left(u_{s}\right)$ is the large-scale envelope of all small-scale fluctuations obtained by first extracting the envelope of the small-scales $\mathcal{E}\left(u_{s}\right)$ by a Hilbert transform procedure as described in Mathis et al, ${ }^{3}$ and then long-wavelength-pass filtering the envelope through the same spatial Fourier filter with cut-off at $5 \delta . R_{a m}$ is simply the correlation coefficient between $u_{l}$ and $\mathcal{E}_{l}\left(u_{s}\right)$, and can also be interpreted as a phase relationship. Figure 6 shows $R_{a m}$ for the canonical and the forced boundary layer at $x / \delta_{1}=2.7$. A good agreement in $R_{a m}$ is found between the canonical flow and earlier studies reported in Mathis et $a l^{3}{ }^{3}$ where $R_{a m}$ shifts gradually from a positive value near to the wall to a negative value approaching the boundary layer edge. This indicates that the small-scale envelope is closer to being in phase with the large-scale motions near to the wall and out of phase at the boundary layer edge.

In comparison with the canonical flow, where only natural large-scales are present, the forced flow shows a broadly similar trend. However, in presence of the synthetic large-scale due to dynamic forcing, $R_{a m}$ is found to increase in the region $0.01<y / \delta<0.1$ and decrease in the region $0.1<y / \delta<0.4$ relative to the canonical flow. This suggests that the synthetic large-scale is driving the small-scale envelope towards being in phase with it below $y / \delta=0.1$ and out of phase with it above $y / \delta=0.1$. It is important to note that there is a good correspondence between regions of increased $R_{a m}$ and $R_{c c}=1$, and regions of decreased $R_{a m}$ and $R_{c c}=-1$. Hence, the modified phase relationship suggested by a change in $R_{a m}$ in presence of the synthetic large-scale is consistent with the influence of the synthetic large-scale on the envelope of triadically interacting small-scales.

Overall, this analysis strongly hints at a phase organization effect on small-scales due to excitation of a synthetic large-scale in the flow. The effect is clearly seen on the small-scales that directly interact with the synthetic large-scale, and is also visible when all the small-scales in the flow are considered.

Finally, we consider the skewness profiles $S k(y)$.. of velocity fluctuations for the canonical and the forced turbulent boundary layer (figure 7). Schlatter and Örlu ${ }^{11}$ show that the amplitude modulation coefficient is closely related to the skewness of velocity fluctuations. As expected, the skewness profiles in figure 7 suggest the same modified phase relationship as the amplitude modulation coefficient.

\section{Summary and conclusions}

In this study, a zero-pressure-gradient turbulent boundary layer flow over a smooth flat plate at $R e_{\tau} \approx 940$ was perturbed using a spatially-impulsive patch of dynamic roughness at the wall following the technique established in Jacobi and McKeon. ${ }^{5}$ The roughness geometry and actuation frequency were chosen to minimize non-equilibrium behavior associated with static roughness effects, and excite a streamwise wavelength in the flow close to the streamwise wavelength of VLSMs observed at higher Reynolds numbers. A phase organizing effect on the small-scales was seen in presence of the synthetic large-scale. This approach allows us to better understand the influence of large-scale motions on the near-wall flow dynamics and explore the possibility of utilizing such synthetic large-scales to manipulate turbulence in a favorable way.

The support of AFOSR (grant FA 9550-12-1-0469, program manager D. Smith) and a Resnick Sustainability Institute Graduate Research Fellowship (S.D.) is gratefully acknowledged.

\section{References}

\footnotetext{
${ }^{1}$ A. J. Smits, B. J. McKeon and I. Marusic, "High Reynolds number wall turbulence", Ann. Rev. Fluid Mech.(2011), vol. 43, pp. 353-375.

${ }^{2}$ N. Hutchins and I. Marusic, "Large-scale influences in near-wall turbulence", Phil. Trans. R. Soc. A (2007), vol. 365, pp. 647-664.

${ }^{3}$ R. Mathis, N. Hutchins and I. Marusic, "Large-scale amplitude modulation of the small-scale structures in turbulent boundary layers", J. Fluid Mech. (2009), vol. 628, pp. 311-337.

${ }^{4}$ I. Jacobi and B. J. McKeon, "New perspectives on the impulsive roughness-perturbation of a turbulent boundary layer", J. Fluid Mech. (2011), vol. 677, pp. 179-203.

${ }^{5}$ I. Jacobi and B. J. McKeon, "Dynamic roughness perturbation of a turbulent boundary layer", J. Fluid Mech. (2011), vol. 688 , pp. $258-296$.
} 
${ }^{6}$ H. M. Nagib, K. A. Chauhan and P. A. Monkewitz, "Approach to an asymptotic state for zero pressure gradient turbulent boundary layers", Phil. Trans. R. Soc. A (2007), vol. 365, pp. 755-770.

${ }^{7}$ D. B. DeGraaff and J. K. Eaton, "Reynolds-number scaling of the flat-plate turbulent boundary layer", J. Fluid Mech. (2000), vol. 422, pp. 319-346.

${ }^{8}$ A. K. M. F. Hussain and W. C. Reynolds, "The mechanics of an organized wave in turbulent shear flow", J. Fluid Mech. (1970), vol. 41-2, pp. 241-258.

${ }^{9}$ D. Chung and B. J. McKeon, "Large-eddy simulation of large-scale structures in long channel flow", J. Fluid Mech. (2010), vol. 661, pp. 341-364.

${ }^{10} \mathrm{I}$. Jacobi and B. J. McKeon, "Phase relationships between large and small scales in the turbulent boundary layer", Exp. Fluids (2013), vol. 54, 1481.

${ }^{11} \mathrm{P}$. Schlatter and R. Örlu, "Quantifying the interaction between large and small scales in wall-bounded turbulent flows: A note of caution", Phys. Fluids (2010), vol. 22, 051704.

10 of 10 